
\title{
25 Research Soure \\ Uterine Rupture of a Patient With Rudimentary Horn Pregnancy at 26th Gestational Weeks Diagnosed via Magnetic Resonance Imaging: a Case Report
}

\section{SEVCAN SARIKAYA}

Mardin State Hospital

MUHSIN NUH AYBAY ( $\sim$ muhsinnuhaybay@gmail.com )

Private Doruk Yildirim Hospital https://orcid.org/0000-0001-5999-6146

\section{Research Article}

Keywords: Müllerian anomaly, Rudimentary horn pregnancy, Rupture, Magnetic Resonance Imaging

Posted Date: January 3rd, 2022

DOl: https://doi.org/10.21203/rs.3.rs-925100/v1

License: (c) (1) This work is licensed under a Creative Commons Attribution 4.0 International License.

Read Full License 


\section{Abstract}

Objective: Pregnancy of Rudimentary Horn is a type of ectopic pregnancy, that is recognized almost always during surgical treatment of a rupture of the rudimentary horn. This is an obstetric case diagnosed preoperatively by magnetic resonance imaging (MRI).

Case: We report the case of a 19-years-old primigravida patient with rupture of rudimentary horn in 26th gestational week. The patient presented with sudden onset severe abdominal pain in the emergency room. Intraabdominal free fluid is detected. To evaluate the etiology of free fluid and location of the gestational sac, an abdominal magnetic resonance imaging (MRI) scan was planned. The decision of emergent laparotomy is made because of sonographic detection of abdominal excessive -concentratedfree fluid, abdominal tenderness, and 2 points decrease of hemoglobin value in the control hemogram. A rudimentary horn pregnancy and fundal rupture of the rudimentary part of the uterus are diagnosed during the surgical procedure. A baby -live- weighing 450 grams was delivered. The ruptured rudimentary horn and same-sided tuba uterina were surgically removed.

Conclusion: Rudimentary horn pregnancy is a rare ectopic pregnancy. Diagnosis is difficult clinically, even with diagnostic imaging modalities. Identifying both cornuas systematically in all patients increases the detection rate. The absence of continuity between the gestational sac's lumen and the cervical canal on imaging is an important finding. Due to the serious maternal and fetal complications, its detection at an early week may be life-saving.

\section{Introduction}

The unicornuate uterus is formed as a result of failure in the development and elongation of the müllerian duct during embryogenesis. As a result of this defect, it usually results in a functional uterus, normal cervix, and fallopian tube with agenesis on the contralateral side or an abnormal müllerian development such as a rudimentary horn (1).

The majority of patients are asymptomatic. The rudimentary horn may or may not be connected with the normal cavity. It may have functional endometrial cavity (2). Pregnancy is rare in the rudimentary horn and is a form of ectopic pregnancy with an incidence of between 1/76.000 to 1/140.000 (3). Despite advances in imaging methods, pregnancy may progress before diagnosis. Pregnancy in rudimentary horns can reach different gestational weeks in patients, depending on the muscular structures of the rudimentary horns. The patient usually applies to the hospital with clinical symptoms of abdominal hemorrhage and acute abdominal pain due to rupture of the uterine wall in the second trimester.

In this case report, a pregnancy that developed in a non-communicating rudimentary horn and resulted in rupture in the late 2 nd trimester is presented.

\section{Case Presentation}


A 19-year-old patient with gravida 2 abortion 1 para 0 came to routine pregnancy controls and was referred last week - because of smaller femur length according to gestational week- from a peripheral hospital to perinatalogy department for Level 2 Ultrasound Scan. Level 2 Ultrasonography of the patient is reported as no skeletal dysplasia was detected, $45 \times 50 \mathrm{~mm}$ submucous myoma uteri was detected in the lower uterine segment. One week later the patient was applied to the emergency room with complaints of abdominal pain, nausea, and vomiting.

As a result of detection of guarding and rebound tenderness in the abdomen during the abdominal examination of the patient, abdominal sonographic examination was performed by Radiology Department for the pre-diagnosis of acute appendicitis. Appendix vermiformis was in normal appearance. There was -predominantly in lower quadrants- excessive free fluid in the abdominal cavity. A fetus compatible with BPD 25 weeks and 2 days- with positive cardiac activity is detected in the gestational sac in the pelvic area. Except for the gestational sac, a left-deviated uterine structure -related to the cervix and vagina- was detected in the pelvic area. There was no connection between the gestational sac and the cervix. To evaluate the etiology of free fluid and location of the gestational sac, an abdominal magnetic resonance imaging (MRI) scan was planned. By the evaluation in comparison with current MRI scan without contrast agent (Fig. 1) and pre-pregnancy (dated 05.03.2020) abdomen MRI scan with contrast agent (Fig. 2); it is understood that there is an anomaly compatible with unicornuate uterus with a rudimentary horn and current 2 nd-trimester gestational sac is located in this rudimentary horn (ectopic pregnancy). Findings were evaluated in favor of a rupture of ectopic pregnancy.

Because the patient had an abdominal tenderness in the abdominal examination, diffuse free fluid in the abdominal cavity; an emergency laparotomy was planned for the patient with the preparation of blood products (first hemoglobin value was $11,2 \mathrm{mg} / \mathrm{dl}$, one hour later was $9,1 \mathrm{mg} / \mathrm{dl}$ ). During the laparotomy in the abdomen appr. $1000 \mathrm{cc}$ of hemorrhagic fluid and coagulated blood was obtained. It was detected that the pregnancy was placed in the rudimentary horn and was ruptured from the left upper wall of the fundus (Fig. 3A). By performing the hysterectomy on the rudimentary uterus, a live fetus was delivered and given to the pediatrician in the operation room. It was observed that the placenta was completely invaded to the rudimentary horn myometrium, the placenta and its appendages could not be separated from the myometrium (placenta percreta) (Fig. 3B). The diagnosis of placenta percreta is confirmed histopathologically. The right rudimentary horn and its fallopian tube were surgically removed. The ureter of the same side was checked due to the high probability of accompanied urinary tract anomalies. Endometriotic foci -probably due to the retrograde menstruation- were observed on Douglas and peritoneal surfaces.

After controlling for intraabdominal bleeding, the abdominal cavity was irrigated with appr. $1000 \mathrm{cc}$ of warm saline. The operation is completed after the replacement of drainage in the abdomen. The abdominal layers were closed. After the closure of abdomen, the patient was placed in lithotomy position and uterine cavity -that associated with cervix- was evacuated with vacuum curettage. During the operation 1 unit of erythrocyte suspension and 1 unit of fresh frozen plasma were infused to the patient. During the postoperative follow-up, a total of $150 \mathrm{cc}$ sero-hemorrhagic fluid drainage was observed. Due 
to the low hemoglobin value and symptoms of anemia by the patient, 2 units of erythrocyte suspension and 1 unit of fresh frozen plasma were infused to the patient. On the 2nd postoperative day, drainage was withdrawn. The patient was discharged on the postoperative 3rd day without any complication.

\section{Discussion}

Unicornuate uterus is associated with appr. $90 \%$ non-communicating rudimentary horn (2). In the uterus with rudimentary horns, the rudimentary part is usually located on the right side. The reason for this is that the left müllerian canal moves caudally than right part (4). In our case too, the rudimentary horn was located on the the right. The rudimentary horn, which is not connected to the cavity-containing uterus, can cause retrograde menstruation, causing hemosalpinx and endometriosis from the same-sided fallopian tubes (5). In our case, endometriotic foci were observed on the pouch of Douglas and peritoneum. The majority of rudimentary horn pregnancy patients were admitted to the hospital -between the gestational weeks of 10th and 20th- with acute abdominal pain as a result of uterine rupture (6). In our case too, rudimentary horn pregnancy could not be diagnosed during routine follow-up and routine secondtrimester ultrasonoghraphic scan, and the patient was admitted to the hospital with acute abdominal pain at 26th gestational weeks. The rudimentary horn was diagnosed by comparing the pre-operative and pre-pregnancy MRI scans, and the diagnosis was confirmed during the surgical procedure.

The rudimentary horn is hard to identify, especially if not suspected, and may be misdiagnosed as pelvic mass or cervix (7). When pregnancy develops in a rudimentary horn, the absence of continuity between the gestational sac's lumen and the cervical canal on ultrasound is an important finding (8).

MRI allows accurate classification of unicornuate uterus. At MRI, the small, curved unicornuate uterus is typically displaced off midline. This appearance is named "Banana" configuration. It has normal myometrial zonal anatomy, with normal endometrial-to-myometrial width and ratio. The appearance of the rudimentary horn varies by subtype. If there is no endometrium present, zonal anatomy is absent and the entire horn may demonstrate diffuse low signal intensity. A rudimentary horn without endometrium and the absent rudimentary horn subtype present minimal risk and do not usually require surgical intervention. However, the presence of endometrium in a rudimentary horn is an important finding and should be reported. A non-communicating rudimentary horn with endometrium may manifest as a large uterine mass, endometriosis, and ectopic pregnancy. If endometrial tissue is present, there may be preserved zonal anatomy. After gadolinium injection; dominant and rudimentary horns show normal myometrial enhancement (9).

In our case, in pre-pregnancy ultrasound and MRI examinations; the rudimentary horn was followed with misdiagnosis as myoma. When we re-evaluated the old MRI images, rudimentary horn and zonal anatomy were clearly observed, especially in the coronal FS T2WI sequence (Fig. 2D). During pregnancy follow-ups, it was not noticed that the gestational sac was not continuous with the cervical canal on ultrasound. Preoperative MRI images revealed that the gestational sac was located separately from the normal uterine cavity (Fig. 1). 
During surgery, same-sided salpingectomy -to avoid following ectopic pregnancies-, excising of the rudimentary horn is recommended. Oophorectomy is not recommended. Laparoscopy can be performed in non-ruptured cases (10). However laparotomy was preferred because our patient was with rupturedrudimentary horn, was in an advanced gestational week, and was hemodynamically unstable. In addition, this anomaly is highly associated with - mostly same sided- urinary tract abnormalities (incidence found to be 36 percent) (11). The incidence of major renal anomalies associated with incomplete uterine duplication with non-communicating rudimentary horn varies between $31 \%$ and $100 \%$. The most common anomaly is renal agenesis on the same side with non-communicating rudimentary horn, while the same-sided pelvic kidney is 2 nd most common one. In our case, no urinary tract anomalies were found.

\section{Conclusion}

Rudimentary horn pregnancy is a rare ectopic pregnancy. Diagnosis is difficult clinically, even with diagnostic imaging modalities. Identifying both cornuas systematically in all patients increases the detection rate. The absence of continuity between the gestational sac's lumen and the cervical canal on imaging is an important finding. Due to the serious maternal and fetal complications, its detection at an early week may be life-saving.

\section{Abbreviations}

MRI, magnetic resonance imaging;

\section{Declarations}

\section{Data and materials availability}

All data were presented in the paper.

\section{Author contribution}

MNA conducted the literature review. SS analyzed and interpreted the patient data for the case presentation. MNA and SS wrote the manuscript together. All authors read and approved the final manuscript.

\section{Ethics approval and consent to participate}

Consent to participate was provided and it may be provided if requested.

\section{Consent for publication}

Consent for publication was provided by the patient and it may be provided if requested. 


\section{Conflict of interest}

The authors declare no competing interests.

\section{References}

1. Nahum GG. Uterine anomallies: how common are they, and what is their distribution among subtypes? J Reprod Med 1998; 43:877-887.

2. Speroff L, Glass RH, Kase Nathan GN. Clinical Gynecologic Endocrinology and Infertility. 1999 pp 123-158.

3. Tsafrir A, Rojansky N, Sela HY, Gomori MJ, Nadjari M. Rudimentary Horn Pregnancy. J Ultrasound Med. 2005; 24: 219-223.

4. Nahum GG: Rudimentary uterine horn pregnancy: the 20th-century worldwide experience of 588 cases. J Reprod Med. 2002, 47:151-163.

5. Nezhat CR, Smith KS. Laparoscopic management of a unicorniate uterus with two cavitated, noncommunicating rudimentary horns. Human Reproduction 1991; 4 (8): 1965- 1968.

6. Edelman AB, Jensen JT, Lee DM, Nichols MD: Successful medical abortion of a pregnancy within a noncommunicating rudimentary uterine horn. Am J Obstet Gynecol. 2003, 189:886-887. 10.1067/S0002-9378(03)00121-2

7. Dove CK, Harvey SM, Spalluto LB: Sonographic findings of early pregnancy in the rudimentary horn of a unicornuate uterus: a two case report. Clin Imaging. 2018, 47:25-29. 10.1016/j.clinimag.2017.08.005

8. Tsafrir A, Rojansky N, Sela HY, Gomori JM, Nadjari M. Rudimentary horn pregnancy: First-trimester prerupture sonographic diagnosis and confirmation by magnetic resonance imaging. J Ultrasound Med. 2005;24(2):219-23.

9. Behr, S. C., Courtier, J. L., \& Qayyum, A. (2012). Imaging of müllerian duct anomalies. Radiographics, 32(6), E233-E250.

10. Dicker D, Nitke S, Shoenfeld A, Fish B, Meizner I, Ben-Rafael Z: Laparoscopic management of rudimentary horn pregnancy. Hum Reprod. 1998, 13:2643-2644. 10.1093/humrep/13.9.2643

11. Jayasinghe $Y$, Rane A, Stalewski H, Grover S: The presentation and early diagnosis of the rudimentary uterine horn. Obstet Gynecol. 2005, 105:1456-1467.

10.1097/01.AOG.0000161321.94364.56

\section{Figures}



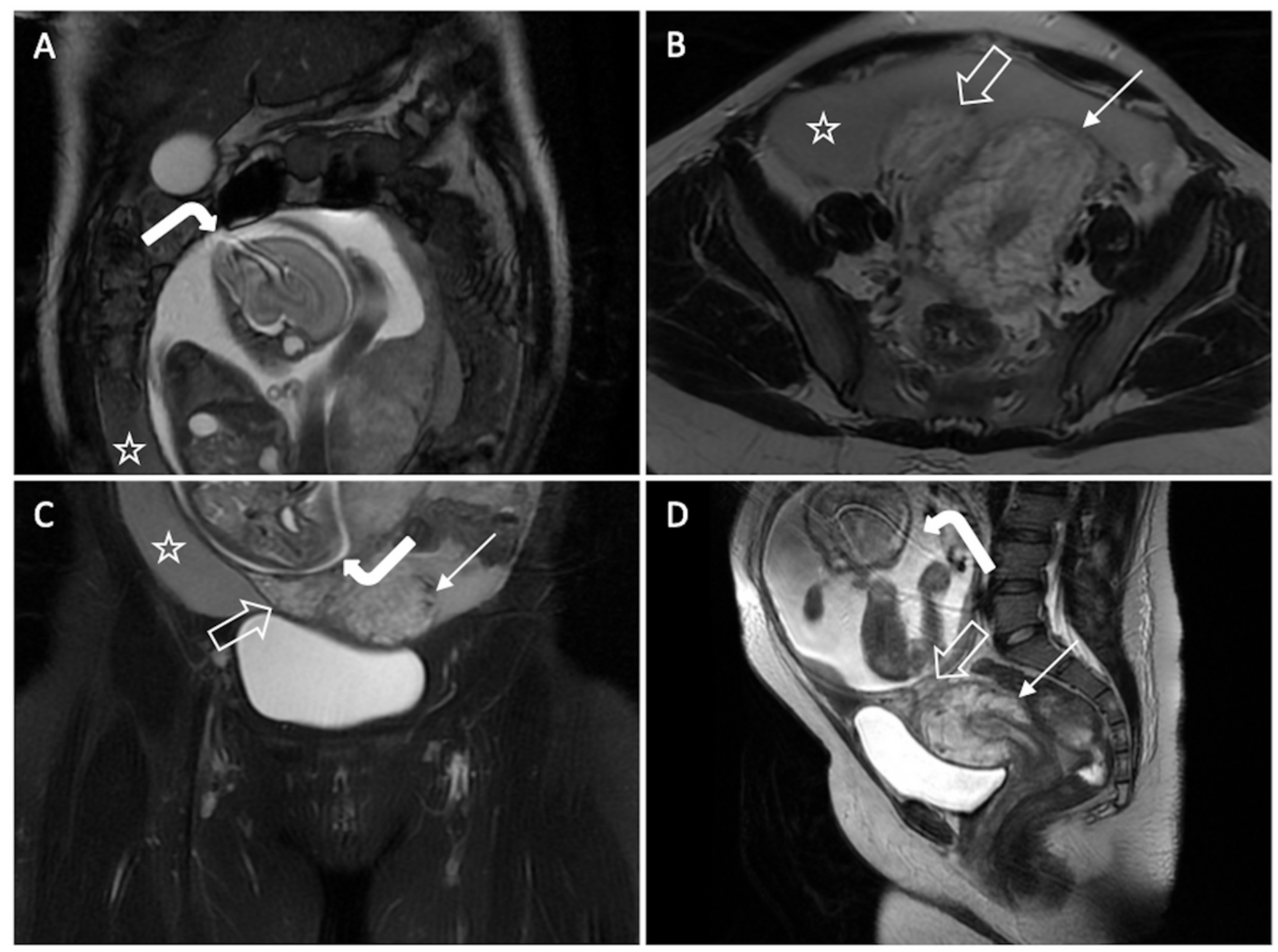

\section{Figure 1}

Coronal (A, C), axial (B), and sagittal (D) T2WI MRI show a large amount of intraperitoneal hemorrhage (stars), a fetus (curved arrows). There was suspected to have a unicornuate uterus that shows a single banana-shaped left uterine horn (thin arrows). There is also a right side pelvic structure located between the uterine fundus and gestational sac (thick open arrows). Note the structure is the same intensity as the myometrium. 

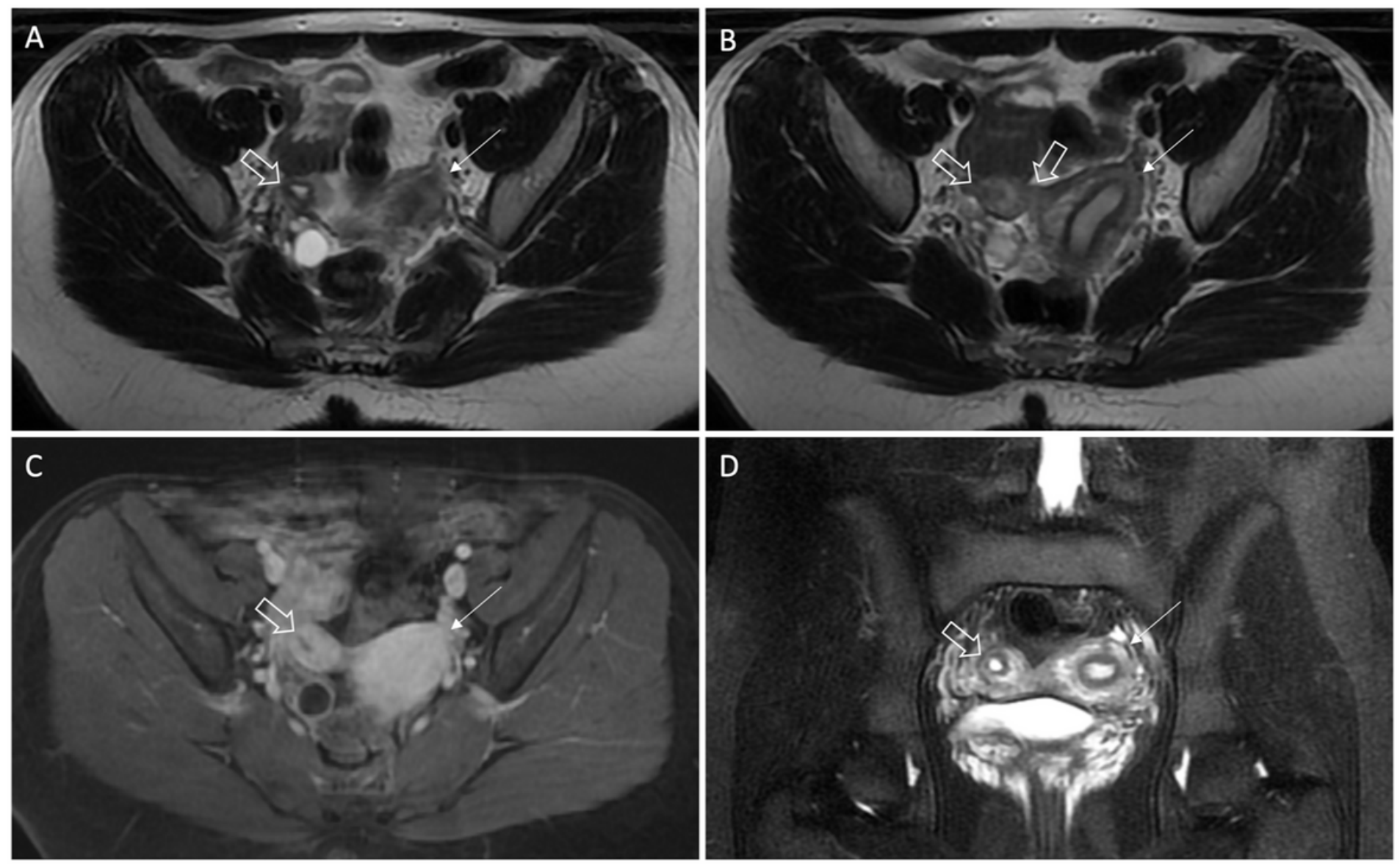

Figure 2

Axial T2WI MRI (A, B), axial contrast-enhanced FS T1WI MRI (B), and coronal FS T2WI MRI (D) show a normal-appearing left uterine horn (thin arrows) and a right side pelvic structure connected with the myometrium (thick open arrows). This is a rudimentary horn that contains zonal anatomy and endometrium. Axial T1WI C+ FS MRI (C); dominant and rudimentary horns show normal myometrial enhancement. 

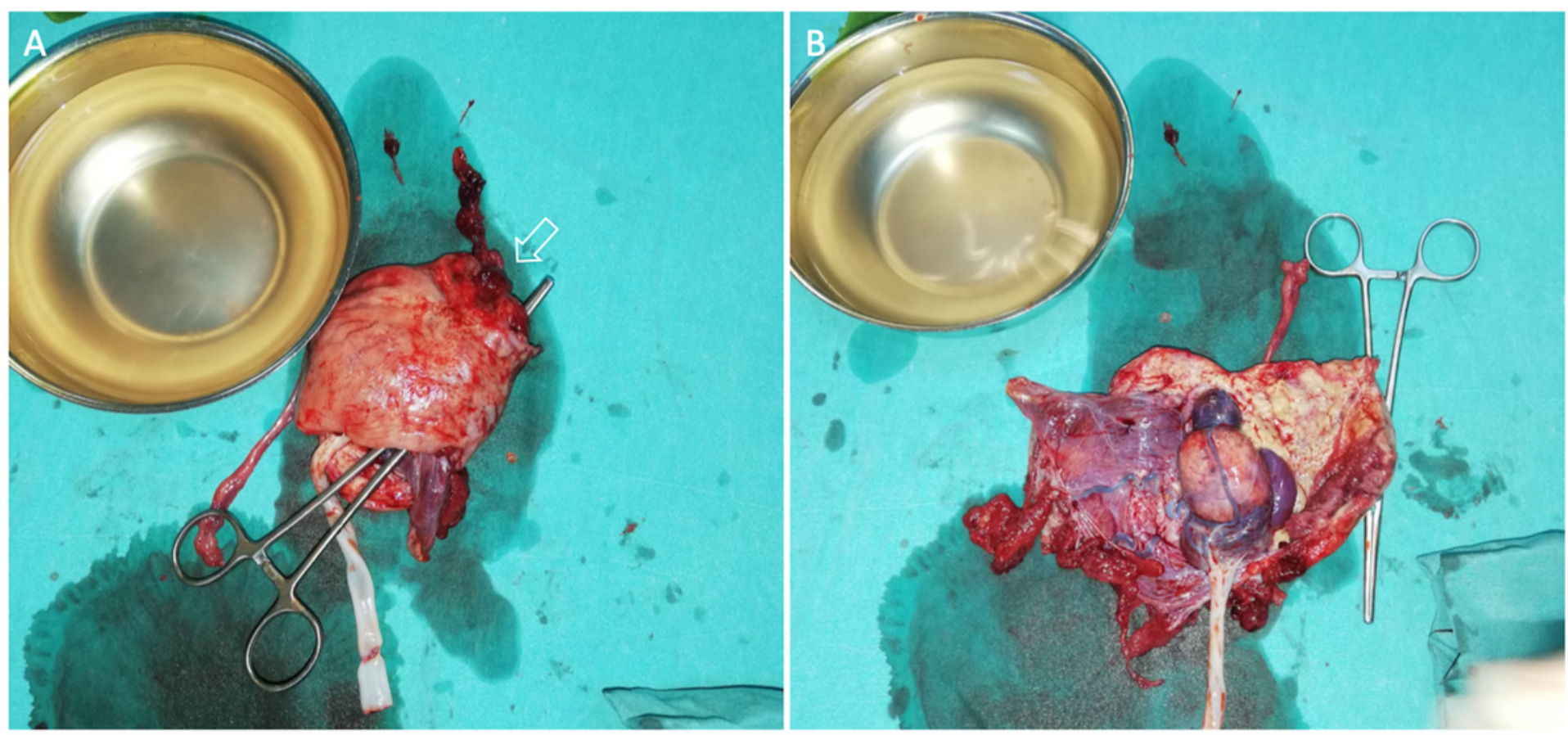

Figure 3

Macroscopic specimen of ruptured (arrow) rudimentary horn (A) and placenta adhesion anomaly area (B)

\section{Supplementary Files}

This is a list of supplementary files associated with this preprint. Click to download.

- CAREChecklist.pdf 